\title{
3D eclipse mapping
}

\section{R.G.M. Rutten}

Netherlands Foundation for Research in Astronomy, Isaac Newton Group of Telescopes, Apartado de Correos 321 , E-38700 Santa Cruz de la Palma, Spain

e-mail: rgmr@ing.iac.es

Received January 24; accepted May 27, 1997

\begin{abstract}
A light curve modelling and fitting program is presented for cataclysmic variable stars and related objects. It allows modelling of a three-dimensional disk in conjunction with the Roche-lobe filling secondary star, thus rendering it a very general tool for light curve analysis. Optimization of the solution is based on the maximumentropy algorithm. Some bench-mark tests are presented, and the general applicability of the program is explored. The program is used here to study the problem of reconstructing the brightness distribution on flaring accretion disks from eclipse light curves, and the results are compared with those obtained using the well-established standard technique of flat-disk eclipse mapping. A previous study suggested that erroneous radial temperature profiles were obtained with flat-disk eclipse mapping. It is shown here that flat-disk eclipse mapping does reproduce the radial brightness structure of flared accretion disks very well, provided that the inner disk is not obscured by the outer rim of the disk.
\end{abstract}

Key words: binaries: eclipsing - cataclysmic variables - accretion

\section{Introduction}

In the study of accretion disks in cataclysmic variable stars (CVs), the analysis of light curves plays an important role. In particular the analysis of eclipse light curves provides information on the disk's brightness structure on scales as small $10^{-6}$ arcseconds that will probably never be resolved by direct imaging techniques. By far the most powerful technique for detailed eclipse light curve analysis is called eclipse mapping, developed by Horne (1985). Classical model fitting techniques, as opposed to reconstruction techniques such as eclipse mapping, dictate the intensity structure on the accretion disk to obey some functional shape. Eclipse mapping, on the other hand, treats the accretion disk as being composed of a large number of elements. The brightness values of these elements are adjusted independently to fit the light curve, without the need to enforce some functional dependence. The only information that is required to reconstruct a brightness map of the surface of the accretion disk is the geometry of the binary system, from which the visibility of each part of the disk at any time during the orbital phase is deduced. The maximum-entropy optimization scheme is used to obtain the smoothest possible brightness distribution.

After several years of experience, eclipse mapping has now become a well established tool (see the reviews by Horne 1993 and Warner 1995). Eclipse mapping relies on a number of basic assumptions, such as the shape and brightness of the secondary star (which is usually assumed to be dark and filling its Roche lobe), and the shape of the accretion disk (which is usually assumed to be geometrically thin, and flat). Some of these model assumptions can in principle be changed. For example, the brightness of the secondary star has been included in models (Rutten et al. 1994), and some have incorporated limited three-dimensional disk shapes (such as flaring disks and disk rims; Wood et al. 1991; Rutten et al. 1992; Billington 1996).

In this paper, a general-purpose 3D light curve fitting program is presented which not only allows the use of nearly any three-dimensional disk shape, but also incorporates the surface of the Roche-lobe filling secondary star. This generalization allows us to extract information from the complete light curve rather than just the eclipse, as in the case of standard eclipse mapping. It also renders the program applicable to the analysis of a large range of light curves, such as, for example, light curves of geometrically thick disks, tilted disks, ellipsoidal variations, heating by irradiation, or any combination of these.

The problem of reconstructing the brightness distribution of flaring accretion disks from eclipse light curves is studied in Sect. 4 as an example of the use of the 3D light curve program. Doubt has been raised in the literature as to whether the radial temperature profile of accretion disks, deduced using the standard flat-disk eclipse mapping technique, is correct. Smak (1994) suggested that 
the flat radial dependence of the effective temperature observed in the disks of some systems (e.g. Rutten et al. 1992; Wood et al. 1992) is due to the fact that eclipse mapping does not take the correct three dimensional disk shape into account. This particular problem will be explored in some detail here by using the standard eclipse mapping method to fit light curves that are calculated for flared disks with a known brightness distribution, and comparing the reconstructed disks' brightness distribution with the original one.

\section{Light curve modelling and fitting}

The light curve mapping program presented here allows one to model a light curve of a cataclysmic variable star, or any related object. The model comprises an accretion disk and a Roche-lobe filling secondary star. The disk can assume nearly any three-dimensional shape. The surface of the disk and the star are approximated by a grid of tiles. Given some combination of orbital inclination and stellar masses, ray-tracing determines what fraction of each grid element is visible at any orbital phase. Each element is assigned an intensity value. The total brightness observed from the system is the weighted sum of intensities, $F_{i}$, of all individual elements, $i$. The weight includes (a) the fractional visibility of the element, (b) its surface area, (c) the fore-shortening projection factor onto the plane of view, and (d) a limb darkening factor (optional; limb darkening may assume its quadratic or linear approximation, or any other functional dependence; see Wade \& Rucinsky (1985) for limb darkening coefficients for stars, and Diaz et al. (1996) for accretion disks).

The light curve, $B(\phi)$, is derived by conducting this summation as a function of orbital phase $\phi$. The visibility matrix $W_{i}(\phi)$ accounts for the weight factors for all grid elements, and as a function of orbital phase. Thus,

$B(\phi)=\sum_{i=1}^{n} W_{i}(\phi) \cdot F_{i}$.

By comparing the calculated light curve for some grid brightness distribution with the measured light curve, the intensities $F_{i}$ of the grid elements can be adjusted iteratively until the measured light curve is optimally fitted. Within the uncertainty on the light curve, however, many different solution are possible that fit the light curve equally well. In order to obtain the smoothest possible, unique solution which fits the data to a reduced $\chi^{2}$ of unity an additional constraint is needed. This is found in the so-called entropy function which is maximized. The maximum-entropy solution to some problem may be looked upon as the most likely solution out of the ensemble of all possible solutions.

The entropy value of an image is measured relative to some default image. The final maximum-entropy solution will tend towards the default image as much as the constraint imposed by the light curve and the associated uncertainties will allow. Following Horne (1985, who pioneered the application of the maximum-entropy criterion in the analysis of eclipse light curves in $\mathrm{CVs}$ ), the default image for the accretion disk is taken to be the azimuthally averaged map of the solution itself. For the surface of the secondary star the default image is taken to be a straight average of all grid intensities. This choice of the default image optimises the solution for studying radial brightness structures on the disk, and it tends to supress structure on the stellar surface. More details on maximum-entropy optimization may be found in, for instance, Burch et al. (1983), Skilling \& Brian (1984), and Skilling \& Gull (1985).

In this light curve fitting technique the basic assumptions are that the secondary star fills its Roche lobe, the geometry of the binary system and the three-dimensional shape of the accretion disk is defined, and the orbital inclination is known. Furthermore, the intensity distribution with respect to the co-rotating frame must not change with time.

The grid consists of a large number of triangular tiles which together shape the surface of the accretion disk and the Roche-lobe filling star. The disk surface is build up around a Cartesian grid in the orbital plane. A third orthogonal coordinate is added to each point in the plane to define the disk's three-dimensional shape. The disk grid possesses a top and bottom surface, which allows for a realistic representation of the disk rim, and also allows accurate modelling of tilted disks. Within the threedimensional shape of the disk, transparent - "optically thin" - surface elements may be defined. An example of a grid structure is shown in Fig. 1.

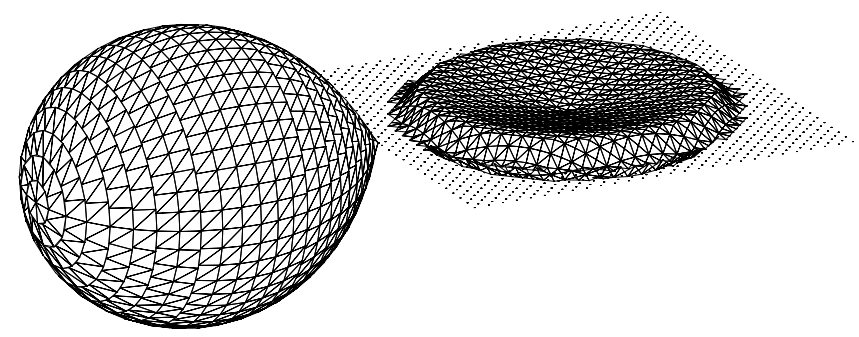

Fig. 1. An example showing the geometry of a grid of surface elements, comprising the secondary star and the accretion disk. The accretion disk is flaring out towards the edge. The mass ratio is unity and the inclination angle is 70 degrees

The fact that the disk may assume some three dimensional geometry aggravates the problem of obtaining a unique solution, in particular in conjunction with the secondary star. A high level of ambiguity in the solution may be present, depending on the exact geometry. Such ambiguity arises when light coming from different parts of 
the binary system have similar impact on the light curve. Bright (or dark) structures on for instance the rim of a thick disk can in some cases be incorrectly interpreted as a brightness structure on the star, since both result in a similar phase-dependent contribution to the light curve. In such situations the light curve can not be used to distinguish between the different brightness structures, and the final solution is defined by the entropy constraint, not by the data. Light curves from eclipsing systems provide the best information on the brightness distribution and allow most accurate distinction between bright structures on the star and the disk. Even more so than in the case of standard eclipse mapping, with 3D light curve fitting results must always be treated with caution, and simulations must be carried out for each choice of geometry in order to understand and eliminate spurious results.

The vast range of possibilites in choice of geometries makes it virtually impossible to characterize the $3 \mathrm{D}$ light curve program in a general sense. The parameter space is too large to describe for all possible geometries how uncertainties on the light curve, or errors on the assumed geometry or on the binary system parameters affect the resulting maps. One qualitative example is presented in the next section. Apart from the flexibility in geometry, this technique is comparable to the standard eclipse mapping technique, and similar limitations and considerations on its use apply (see Horne 1985, 1993).

\section{An example}

A number of controlled experiments have been performed to test the quality of the light curve reconstruction routine described above. First a light curve is calculated assuming some set of binary star parameters and a pre-defined brightness distribution across the disk and the star. Next, the brightness distribution over the grid is reconstructed from the light curve, and the result is compared with the original brightness distribution.

From the very large number of grid geometries and brightness structures that can be studied, only one, but fairly realistic example is presented here. A binary star mass ratio of unity, an orbital inclination of 80 degrees (i.e. an eclipsing system), and an accretion disk with an opening angle of 5 degrees (as measured from the orbital plane) have been assumed. The disk extends out to a radius of 80 percent of the distance to the inner Lagrange point. Its outer rim is rounded off, not only to appear more realistic, but also to reduce jumps in the light curve due to the finite size of surface elements. Note that this definition of the disk surface is rather arbitrary, but suitable for this example. For more realistic descriptions of accretion disk surfaces see Warner (1995), and references therein.

The radial brightness distribution of the disk is based on the standard accretion disk model, with $T_{\text {eff }}^{4} \propto R^{-3}$ for distances from the center of the disk, $R$, large compared to the white dwarf radius (see Frank et al. 1992 for the exact formula). On the secondary star's surface the effects of irradiation by the disk are represented by a luminous inner hemisphere and a dark outer hemisphere.

Figure 2 shows the resulting light curve. Clearly visible are the effects of ellipsoidal variation of the irradiated hemisphere, resulting in large amplitude brightness changes throughout the orbital cycle. Furthermore, a primary eclipse of the disk and a weak secondary eclipse of the star are seen. This light curve is fitted to reconstruct the intensity distribution using the same grid geometry. From the resulting intensity distribution a light curve is constructed and also shown in Fig. 2; it is nearly indistinguishable from the original light curve. The fitted brightness distribution also correlates closely with the input model, as can be seen (qualitatively) from the grey scale representation in Fig. 3, which shows the bright accretion disk with the fainter, thick outer rim, and the bright inner hemisphere of the star. The boundary between the inner and outer hemisphere of the star is not as sharply marked in the reconstruction as in the original input model. How well the original model is reproduced depends mainly on the size of errors that are adopted for the light curve.

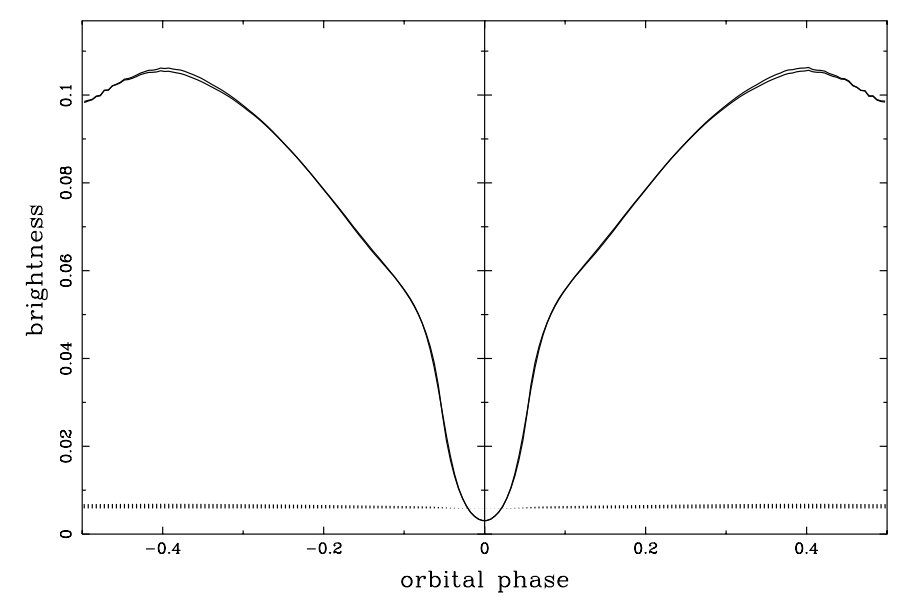

Fig. 2. Light curve of an eclipsing CV where the secondary star contributes strongly to the total light, but only from its inner hemisphere. This crudely mimics irradiation of the secondary star by the accretion disk. The thin curve traces the computed light curve, while the bold curve (nearly indistiguishable at most phases) shows the fit to that light curve. The uncertainties on the light curve as assumed in the fit are shown as vertical bars in the lower part of the plot

As another example of the versatility of the 3D light curve program a light curve is calculated, shown in Fig. 4, for the case of an opaque but thin accretion disk which is tilted relative to the orbital plane. The top of the disk, as seen from the observer, is tilted pointing away from the secondary star. The tilt angle is taken to be 10 degrees, while the orbital inclination is 75 degrees. How the secondary star and the accretion disk each contribute to the 


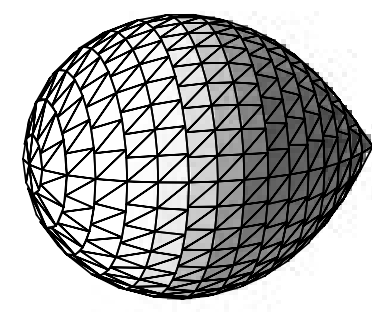

Fig. 3. The intensity structure of the accretion disk and the star as derived from the fit to the light curve shown in Fig. 2. Dark structures in the plot indicate high intensities. Clearly visible is the bright inner hemisphere of the star, and the thick accretion disk with the bright inner disk and the fainter outer rim

light curve is also shown in Fig. 4. The uniformly bright secondary star produces elipsoidal variations. The tilted accretion disk produces an orbital variation due to its changing aspect angle, and it is responsible for a fairly pronounced secondary eclipse because a large portion of the star is obscured. At this orbital inclination the bright center of the accretion disk is just being eclipsed at orbital phase zero.

In the same Fig. 4 the light curve is also plotted for the situation where the disk is assumed optically thin. In that case there are no orbital variations from the tilted disk, and the primary eclipse is much more pronounced. Such comparison offers a way to gauge the optical thickness of accretion disks observationally.

As a final example a model light curve is shown in Fig. 5 for an eclipsing $\mathrm{CV}$ consisting of a thick disk with a bright spot on its outer rim, and a bright white dwarf. These features result in an orbital hump, and in the deep eclipse of the white dwarf and of the bright spot that are so well known from dwarf novae in quiescence.

\section{Eclipse mapping of flared disks}

Smak (1994) studied the problem of the reconstruction of the radial temperature distribution of accretion disks from eclipse light curves. His study suggested that particularly at the highest inclinations flat radial temperature profiles were obtained with eclipse mapping. He concluded that this was an artefact of the standard eclipse mapping technique which incorrectly assumes that the disk is flat and thin, although actually no calculations with the standard eclipse mapping code were used to corroborate that conclusion. The present paper conducts experiments to test how well standard eclipse mapping reproduces the disk radial brightness profile (and thus the radial temperature profile) in the case of flared, thick disks.

First, we define a three-dimensional accretion disk surface with a fixed opening angle and a well-defined outer rim, similar to that shown in Fig. 1. Second, we calculate the brightness distribution of the disk, following the canonical steady-state disk model (see Frank et al. 1992) based on $T_{\text {eff }} \propto R^{-3 / 4}$, and assuming a mass transfer rate of $310^{-9} M_{\odot} /$ year. The secondary star does not contribute to the light curve. Third, the light curve of this flaring disk is calculated by summing intensities from all the visible parts of the disk in the appropriate proportions, as a function of orbital phase (see Sect. 2). Random noise at a level of one percent is added. Finally, the resulting light curve is fitted using the standard thin-disk eclipse mapping method (Horne 1985; Rutten et al. 1992), assuming the correct values for the mass ratio, orbital period and orbital inclination. From the resulting brightness profile the radial temperature profile is calculated and compared with the original temperature profile of the model.

This experiment is conducted for a range of disk opening angles varying from 0 to 15 degrees (as measured from the orbital plane), and orbital inclinations between 70 and 90 degrees. These tests include rather extreme eclipse situations where in some cases the disk is only partly eclipsed, and in other cases the rim of the thick disk obscures the inner disk. Three examples of the reconstructed radial temperature profiles are shown in Fig. 6, two for the case where the disk opening angle is 5 degrees, and an orbital inclination of respectively 80 and 85 degrees, and a third case with an opening angle of 10 degrees viewed a an inclination of 80 degrees. In the latter two cases the front part of the disk surface is just at the verge of being obscured by the disk rim. These examples show good agreement between the reconstructed temperature profile and the theoretical profile for the standard steady-state accretion disk, as indicated by the full curves in Fig. 6. The deviation of the fit from the model in the center of the disk is due to smearing of the flux, which is inherent to the maximum entropy optimization scheme. At the cool and faint outer edge of the disk the fit deviates slightly from the model at the point where the thick, flaring disk is rounded off and edges. These examples are representative for the other reconstructions for different inclinations and disk opening angles, with the exception of situations where the inner disk is completely obscured by the outer disk rim. In the those cases the reconstruction does exhibit a flat temperature structure.

This experiment shows that the standard thin-disk eclipse mapping technique reproduces the radial temperature distribution of accretion disks reasonably accurately, even in cases when the disk is flaring. The reason for the discrepancy between this result and that presented by Smak (1994) is not immediately obvious, except for the fact that that paper did not actually employ a standard maximum entropy eclipse mapping code. The present invesitgation does agree with Smak's work in the case where the disk is thick enough to self obscure its inner parts. In such situation the radial temperature profile can obviously not be reconstructed, since no information on the inner disk is available in the light curve. 


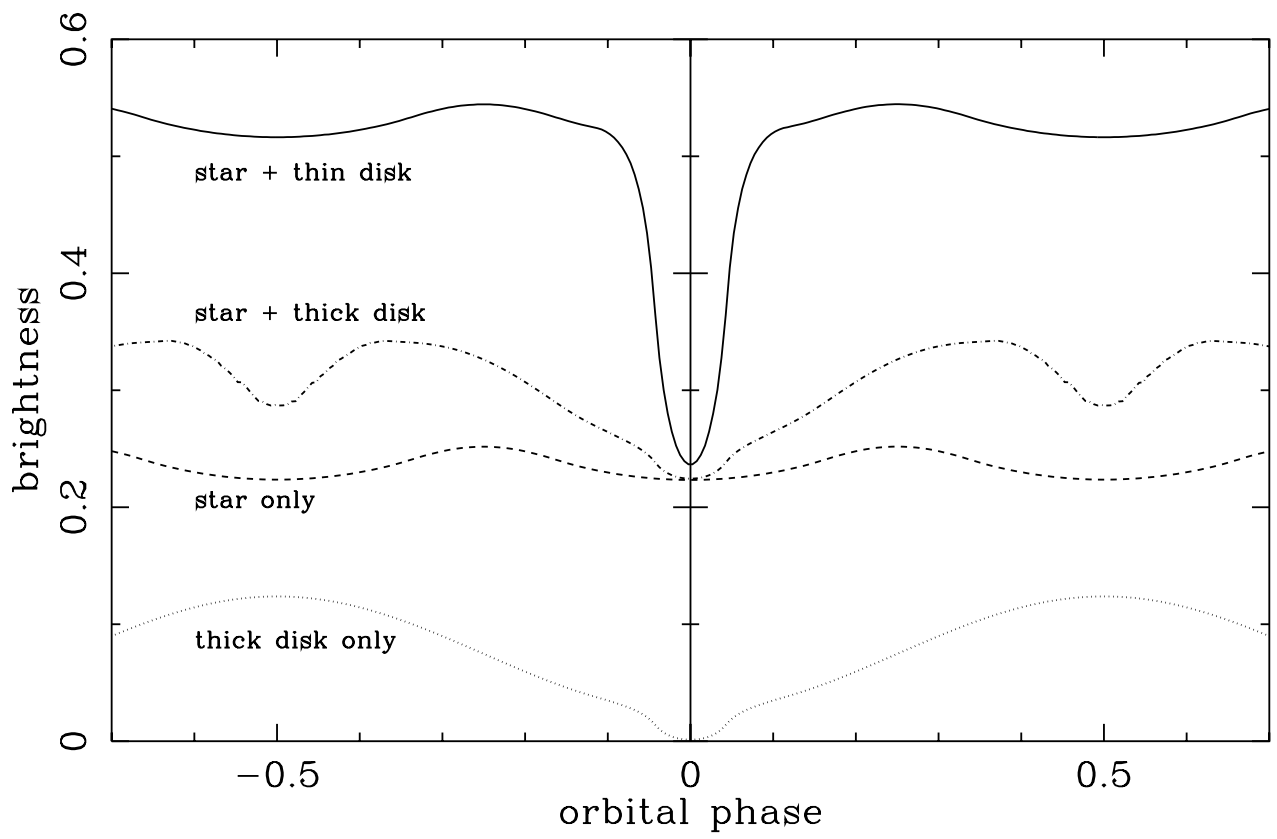

Fig. 4. Model light curve of an eclipsing binary system with a tilted accretion disk. The solid curve shows the case for a transparent disk, while the dash-dotted line traces the light curve for an optically thick disk which obscures the secondary star around phase 0.5 . The dash-dotted and dashed curves separate out the contributions of the secondary star and of the accretion disk respectively

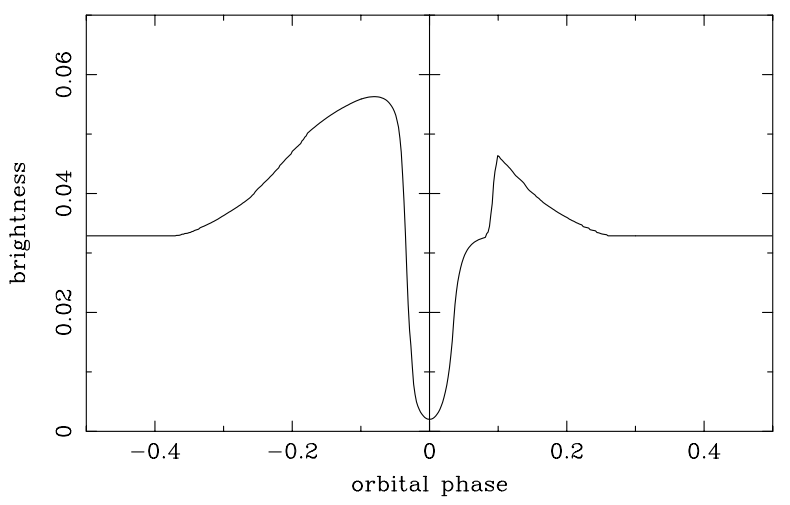

Fig. 5. Model light curve of an eclipsing $\mathrm{CV}$ with a thick accretion disk, a bright spot and a conspicious white dwarf

\section{Conclusions}

The extension of the standard thin, flat disk eclipse mapping algorithm to a truly three-dimensional light curve fitting program provides a useful tool for (i) modelling light curves of nearly any shape of accretion disk and any brightness structure on the disk and the secondary star, and (ii) finding the brightness structure of the three dimensional accretion disk surface and the secondary star by fitting the light curves, accounting for primary and secondary eclipses, or any other change in brightness dur- ing the binary orbit. This algorithm allows a vast range of binary star and accretion disk geometries to be studied, which facilitates easy exploration of various geometries in order to understand observed light curves. However, caution is required when interpreting the results since the large number of possible geometries aggravates the problem of finding a unique and correct solution.

This program was used to construct light curves of flaring disks in eclipsing systems. Fits of these eclipse light curves with the incorrect assumption of a flat and thin disk are shown to still accurately reproduce the disks' radial temperature profile, provided that the inner disk is not obscured by the outer disk rim. This shows that standard eclipse mapping is capable of reproducing the disks' radial temperature profile even in the case of flared disks, contrary to what has been suggested by Smak (1994).

The most important usage of this light curve fitting method is expected to be in situations where both the secondary star and the accretion disk contribute substantially to the light curve, as is often the case at infra-red wavelengths. Also when the disk shape is obviously important, such as for example in high inclination systems, or in the case of tilted or warped disks, this program may serve as a helpful tool in fitting and predicting light curves.

Acknowledgements. I thank Vik Dhillon for many useful discussions, and two referees for valuable comments on the manuscript. 


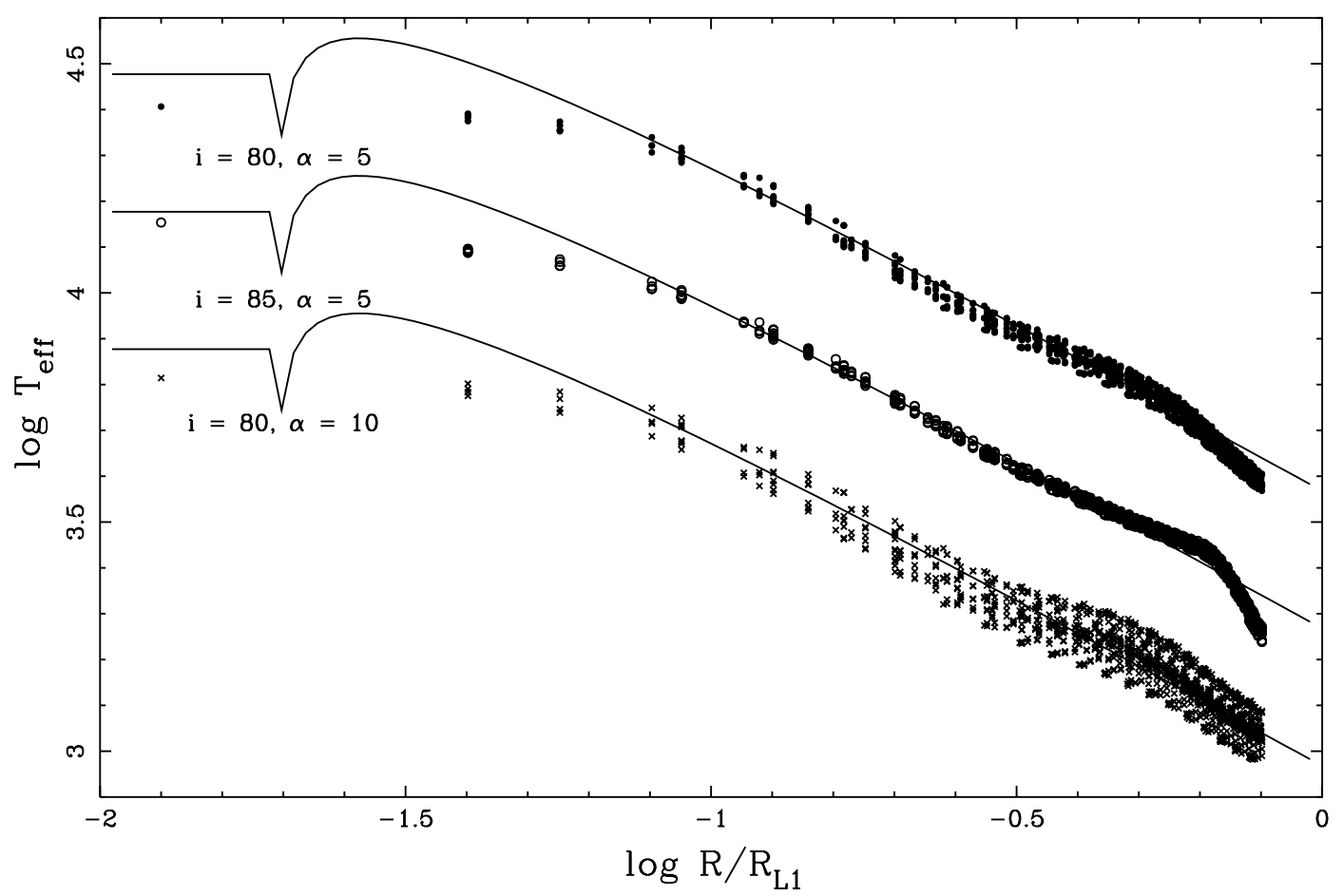

Fig. 6. Three examples of a reconstructed radial temperature profile using standard eclipse mapping for the case of a thick, flared accretion disk. The theoretical profile is shown by the solid curves (note that the lower two curves are displaced by 0.3 and 0.6 dex relative to the top curve for clarity). The orbital inclination and disk opening angle are specified in the diagram

\section{References}

Billington I., Marsh T.R., Horne K., et al., 1996, MN 279, 1274 Burch S.F., Gull S.F., Skilling J., 1983, Computer Vision, Graphics, and Image Processing 23, 113

Diaz M.P., Wade R.A., Hubeny I., 1996, ApJ 459, 236

Frank J., King A., Raine D., 1992, Accretion Power in Astrophysics. Cambridge University Press

Horne K., 1985, MN 213, 129

Horne K., 1993, in Accretion Disks in Compact Stellar Systems, Wheeler J.C. (ed.)

Rutten R.G.M., van Paradijs J., Tinbergen J., 1992, A\&A 260, 213
Rutten R.G.M., Dhillon V.S., Horne K., Kuulkers E., 1994, A\&A 283, 441

Skilling J., Bryan R.K., 1984, MN 211, 111

Skilling J., Gull S.F., 1985, in Maximum-Entropy and Bayesian Methods in Inverse Problems, Smith C.R., Grandy W.T. (eds.). Reidel, Dordrecht, p. 83

Smak J., 1994, Acta Astron. 44, 265

Wade R.A., Rucinsky S.M., 1985, A\&AS 60, 471

Warner B., 1995, Cataclysmic variable Stars. Cambridge University Press

Wood J.H., Abbott T.M.C., Shafter A.W., 1992, ApJ 393, 729 